\title{
UNIFORM APPROXIMATION ON NONCOMPACT SPACES
}

\author{
BY
}

R. E. EDWARDS

Introduction and summary. Throughout this paper, $G$ will denote a Hausdorff locally compact space, subsequently specialized into a Hausdorff locally compact Abelian group. We write $B(G)$ for the Banach space of all bounded, complexvalued functions on $G$ which are universally measurable (i.e., measurable for every Radon measure on $G$ ), the norm being the sup norm. $B C(G)$ denotes the subspace composed of the continuous functions in $B(G)$, whilst $C_{c}(G) \subset B C(G)$ is composed of the continuous functions with compact supports, and $C_{0}(G) \subset B C(G)$ is the closure in $B(G)$ of $C_{c}(G)$. If $G$ is an Abelian topological group, $B U C(G)$ denotes the subspace of $B C(G)$ composed of the bounded uniformly continuous functions on $G$; in this case, $C_{c}(G) \subset C_{0}(G) \subset B U C(G)$. In any case, $M(G)$ denotes the space of all bounded complex Radon measures on $G$, which we will regard as being isometrically injected into the dual of $B(G)$.

Hereafter, the term "measure" will always mean "Radon measure." Concerning (not necessarily bounded) measures on $G$, a net $\left(\mu_{i}\right)$ of such measures will be said to converge vaguely to a measure $\mu$, if and only if

$$
\lim _{i} \int_{G} f d \mu_{i}=\int_{G} f d \mu
$$

for each $f \in C_{c}(G)$. We recall the well-known fact (a consequence of the definition of Radon measures and of a general result in duality theory; see [2, Chapter 4 and Theorem 1.11.4]) that a set $S$ of measures on $G$ is vaguely relatively compact whenever

$$
\operatorname{Sup}\{|\mu|(K): \mu \in S\}<\infty
$$

for each compact subset $K$ of $G$.

In $\$ 1$ we consider the representation of continuous linear functionals on subspaces $V$ of $B(G)$ in a manner suggested by and extending a result of Mazur applying when $G$ is the discrete space of natural numbers, i.e, as limits of integrals with respect to norm-bounded sequences or nets in $M(G)$. Combining this with the Hahn-Banach theorem, we derive some criteria for uniform approximation.

In $\$ 2$ we apply some of these criteria to the case in which $G$ is a Hausdorff

Received by the editors June 13, 1964 and, in revised form, September 10, 1965. 
locally compact Abelian group, focusing attention on the uniform span $T[f]$ of translates of a function $f$ in $B U C(G)$ and its relation to the spectral analysis of $f$. In particular, we examine the dependence of $T[f]$ on variations of $f$ which are small in a certain sense. These results differ from most of those included under the general heading of the spectral theory of such functions, inasmuch as the uniform topology replaces the strict and narrow topologies customarily used (as, for example, in Herz's survey article [1]; see also (3.1, ii) infra).

For functions $f$ which are not uniformly continuous, $T[f]$ is somewhat intractable. For this reason we shall formulate the results in $\S 2$ in terms of $T^{*}[f]$, the uniform closure of the set of functions $K * f$ obtained as $K$ ranges over $L^{1}(G)$. [An alternative would be to allow $K$ to range over $M(G)$.] Notice that $T[f]=T^{*}[f]$ whenever $f \in B U C(G)$; that $T^{*}[f] \subset B U C(G)$ for any $f \in B(G)$; and that $f \in T^{*}[f]$ if and only if $f \in B U C(G)$.

It is also worth remarking that, if $T_{s}[f]$ denotes the strict closure of the set of linear combinations of translates of $f \in B C(G)$, then $T_{s}[f]=$ strict closure of $T^{*}[f]$. This may be seen by a direct application of the Hahn-Banach theorem, combined with the fact that the dual of $B C(G)$ relative to the strict topology may be identified with $M(G)$.

A lengthy section (2.9) is devoted to concrete examples, mainly for $G=R^{n}(n \geqq 1)$.

In $\S 3$, we consider briefly an approach to problems of approximation in the strict and uniform topologies for suitable subspaces of $B C(G)$.

We emphasise finally that the main interest resides in those cases in which $G$ is noncompact. With the exception of the negative results in (2.3), all the results are widely known to simplify and admit strengthening when $G$ is compact.

\section{Representation of functionals.}

(1.1) THEOREM. Let $V$ be a vector subspace of $B(G)$, and let $M$ be a convex and balanced subset of $M(G)$ such that

$$
\|f\| \leqq \operatorname{Sup}_{\mu \in M}\left|\int_{G} f d \mu\right| \quad(f \in V) .
$$

If $F$ is any continuous linear functional on $V$ of norm at most 1 , there exists a net $\left(\mu_{i}\right)$ extracted from $M$ such that

$$
F(f)=\lim _{i} \int_{G} f d \mu_{i} \quad(f \in V) .
$$

If, furthermore, $V$ is separable, the net $\left(\mu_{i}\right)$ may be taken to be a denumerable sequence $\left(\mu_{i}\right)_{i=1}^{\infty}$.

Proof. From (1.1.1) and the assumption $\|F\| \leqq 1$, it follows that

$$
|F(f)| \leqq \operatorname{Sup}_{M}\left|\int_{G} f d \mu\right|
$$


for each $f \in V$. We now appeal to the bipolar theorem [2, Theorem 8.1.5] in order to conclude that $F$ lies in the $\sigma\left(V^{\prime}, V\right)$-closure of $M$. This yields (1.1.2).

If $V$ is separable, the topology $\sigma\left(V^{\prime}, V\right)$ induces on the unit ball of $V^{\prime}$ a metrisable topology, so that $F$ will be the $\sigma\left(V^{\prime}, V\right)$-limit of a denumerable sequence $\left(\mu_{i}\right)_{i=1}^{\infty}$ extracted from $M$. The proof is complete.

For separable subspaces $V$ it is possible to refine somewhat the representation (1.1.2).

(1.2) COROLlaRY. Let Vbe a separable vector subspace of $B(G)$ and $F$ a continuous linear functional on $V$. Then there exists a positive, bounded, Radon measure $\lambda$ on $G$ and a sequence $\left(\phi_{i}\right)_{i=1}^{\infty}$ of functions in $C_{c}(G)$ such that

$$
\operatorname{Sup}_{i} \int_{G}\left|\phi_{i}\right| d \lambda \leqq\|F\|
$$

and

$$
F(f)=\lim _{i} \int_{G} f \phi_{i} d \lambda \quad(f \in V) .
$$

Proof. We may assume that $\|F\|=1$. Then, applying (1.1) with $M$ taken to be the unit ball in $M(G)$, we conclude that there exists a sequence $\left(\mu_{i}\right)_{i=1}^{\infty}$ extracted from $M$ such that (1.1.2) holds. Now put $\lambda=\sum_{i=1}^{\infty} i^{-2}\left|\mu_{i}\right|$, which is a positive element of $M(G)$. By the Lebesgue-Radon-Nikodym theorem, we may write $\mu_{i}$ in the form $\psi_{i} \cdot \lambda$, where $\psi_{i} \in L^{1}(G, \lambda)$ and

$$
\int_{G}\left|\psi_{i}\right| d \lambda=\int_{G} d\left|\mu_{i}\right| \leqq 1 .
$$

Finally, for each $i$ we may select $\phi_{i} \in C_{c}(G)$ such that

$$
\int_{G}\left|\psi_{i}-\phi_{i}\right| d \lambda \leqq i^{-1}, \quad \int_{G}\left|\phi_{i}\right| d \lambda \leqq 1 .
$$

Then, if $f \in V$ one has

$$
\begin{aligned}
\lim _{i} \int_{G} f \phi_{i} d \lambda & =\lim _{i} \int_{G} f \psi_{i} d \lambda \\
& =\lim _{i} \int f d \mu_{i} \\
& =F(f),
\end{aligned}
$$

by (1.1.2), which is the desired representation formula.

(1.3) REMARKs. Corollary (1.2) may be regarded as an analogue and a generalisation of the result of Mazur, applying when $G$ is the discrete space of natural numbers, and given on pp. 71-72 of Banach [3]. 
We shall digress temporarily to show that the "sequential" nature of (1.2) cannot be maintained without some countability restriction on $V$. This may be inferred by combining the following two assertions: let $L$ be the subset of $V^{\prime}$ composed of all linear functionals on $V$ of the type

$$
f \rightarrow \int_{G} f d \mu,
$$

where $\mu \in M(G)$ and $\|\mu\| \leqq 1$; then

(i) if $V$ contains the characteristic functions of all $\sigma$-compact $G_{\delta}$-subsets of $G$, $L$ is sequentially closed in $V^{\prime}$ for the topology $\sigma\left(V^{\prime}, V\right)$;

(ii) $L$ is closed in $V^{\prime}$ for the topology $\sigma\left(V^{\prime}, V\right)$, if and only if $G$ is finite. Accordingly, if $G$ is infinite and $V$ is as in (i), there exist functionals $F$ which are weakly adherent in $V^{\prime}$ to $L$ and yet are not representable by (1.1.2) for any choice of a denumerable sequence $\left(\mu_{i}\right)_{i=1}^{\infty}$.

Proof of (i). Let $\left(\mu_{i}\right)_{i=1}^{\infty}$ be a sequence extracted from the unit ball of $M(G)$ such that

$$
F(f)=\lim _{i} \int_{G} f d \mu_{i}
$$

Let the measure $\lambda$ and the functions $\psi_{i}$ be defined as in the proof of (1.2).

By hypothesis, then,

$$
\lim _{i} \int_{A} \psi_{i} d \lambda
$$

exists finitely for each $\sigma$-compact $G_{\delta}$-set $A \subset G$. Since each $\sigma$-finite $\lambda$-measurable set differs by a $\lambda$-null set from some $\sigma$-compact $G_{\delta}$-set, it follows (see [2, Proposition 4.21.1 and the proof of Theorem 4.21.4]) that the sequence $\left(\psi_{i}\right)_{i=1}^{\infty}$ is weakly convergent to some $\psi \in L^{1}(G, \lambda)$. Plainly,

$$
\int_{G}|\psi| d \lambda \leqq \lim _{i} \inf \int_{G}\left|\psi_{i}\right| d \lambda \leqq 1 .
$$

It follows at once that (1.3.1) holds if we take $\mu=\psi \cdot \lambda$, which belongs to the unit ball of $M(G)$.

We add the remark that, if $V$ is closed in $B(G)$, it must (under the hypothesis in (i)) contain $B C(G) \supset C_{0}(G)$. Then any sequence $\left(\mu_{i}\right)$ extracted from $M(G)$, for which the limit (1.3.2) exists finitely for each $f \in V$, is necessarily norm-bounded (as follows from Corollary 7.1.2 of [2]). In this case, therefore, we may assert that the set of linear functionals on $V$ of the form $f \rightarrow \int_{G} f d \mu$, where $\mu$ ranges over $M(G)$, is itself sequentially closed for $\sigma\left(V^{\prime}, V\right)$.

Proof of (ii). Assume that $L$ is closed for the topology $\sigma\left(V^{\prime}, V\right)$. Let us show first that $G$ must be compact.

Were $G$ noncompact, we could choose a net $\left(x_{i}\right)$ tending to infinity in $G$ and construct the functional 


$$
l_{0}(f)=\operatorname{LIM} f\left(x_{i}\right),
$$

where LIM is a generalized limit on the space of all bounded, complex-valued nets $\left(\xi_{i}\right)$. On the assumption that $L$ is $\sigma\left(V^{\prime}, V\right)$-closed, it follows easily from the bipolar theorem that there would exist a $\mu \in M(G)$ such that

$$
l_{0}(f)=\int_{G} f d \mu \quad(f \in V) .
$$

Since $l_{0}(f)=0$ for any $f \in B(G)$ which vanishes off some compact subset of $G$, it would follow that $\mu(A)=0$ for all relatively compact $G_{\delta}$-sets $A \subset G$, whence it would follow that $\mu=0$. This is, however, absurd since then (1.3.4) would conflict with the fact that $l_{0}(1)=\operatorname{LIM} 1=1$. This contradiction shows that $G$ must indeed be compact.

A closely analogous argument shows that $G$ must be discrete. Discreteness and compactness add up to finiteness, and the proof is complete.

Despite the preceding remarks, it is true and significant that, when $G$ is a group and $V \subset B C(G)$, at least some of the refining features of (1.2) are attainable without assuming separability of $V$. This is the content of the next result.

(1.4) COROLlary. Let $G$ be a Hausdorff locally compact group with left Haar measure $d x, V$ a vector subspace of $B C(G)$, and $F$ a continuous linear functional on $V$. Let $\Phi$ be a convex and balanced subset of $L^{1}(G)$ whose intersection with the unit ball in $L^{1}(G)$ is dense in that unit ball. Then there exists a net $\left(\phi_{i}\right)$ extracted from $\Phi$ such that

$$
\operatorname{Sup}_{i} \int_{G}\left|\phi_{i}\right| d x \leqq\|F\|
$$

and

$$
F(f)=\lim _{i} \int_{G} f \phi_{i} d x \quad(f \in V) .
$$

If $V$ is separable, we may in addition suppose that the net $\left(\phi_{i}\right)$ is a denumerable sequence $\left(\phi_{i}\right)_{i=1}^{\infty}$.

Proof. We may assume that $\|F\|=1$. In Theorem (1.1) we take for $M$ the set of all measures $\mu$ of the type $d \mu(x)=\phi(x) d x$ as $\phi$ ranges over $\Phi$. It is very simple to verify that (1.1.1) is fulfilled, using the fact that $V \subset B C(G)$. An application of Theorem (1.1) then yields the stated conclusion.

By combining the preceding results with the Hahn-Banach theorem we may derive some criteria for uniform approximation. Three examples follow.

(1.5) THEOREM. Let $G$ be a Hausdorff locally compact Abelian group, $f \in B C(G)$, $g \in B C(G)$, and let $\Phi$ be as in (1.4). In order that $g \in T^{*}[f]$, it is necessary and sufficient that 


$$
\lim _{i} \phi_{i} * K * f(0)=0 \quad\left(K \in L^{1}(G)\right)
$$

shall imply

$$
\lim _{i} \phi_{i} * g(0)=0
$$

whenever $\left(\phi_{i}\right)$ is an $L^{1}(G)$-bounded net extracted from $\Phi$.

Proof. It suffices to appeal to Corollary (1.4) after making the trivial change of replacing each $\phi \in \Phi$ by the function $\check{\phi}(x)=\phi(-x)$.

We add that $G$ is assumed to be Abelian only for simplicity; this special case is adequate for the applications to be made in $\$ 2$ infra.

REMARK. If $L^{1}(G)$ is separable, then $T^{*}[f]$ is separable and we may in the statement of Theorem (1.5) assume that $\left(\phi_{i}\right)$ is a denumerable sequence $\left(\phi_{i}\right)_{i=1}^{\infty}$.

There is an analogue of Theorem (1.5) for spans of translates.

(1.5A) THEOREM. Suppose $G, f, g$ and $\Phi$ are as in Theorem (1.5). In order that $g \in T[f]$ it is necessary and sufficient that

shall imply

$$
\lim _{i} \phi_{i} * f(x)=0 \quad(x \in G)
$$

$$
\lim _{i} \phi_{i} * g(0)=0
$$

whenever $\left(\phi_{i}\right)$ is an $L^{1}(G)$-bounded net extracted from $\Phi$.

(1.6) TheOREM. Let $G$ be a Hausdorff locally compact Abelian group, $X$ its character group, $X_{0}$ a subset of $X$, and $f \in B C(G)$. Let $\Phi$ be as in (1.4). In order that $f$ be the uniform limit of linear combinations of characters $\xi \in X_{0}$, it is necessary and sufficient that

$$
\lim _{i} \int_{G} f \phi_{i} d x=0
$$

for any $L^{1}(G)$-bounded net $\left(\phi_{i}\right)$ extracted from $\Phi$ which satisfies

$$
\lim _{i} \hat{\phi}_{i}(\xi)=0 \quad\left(\xi \in X_{0}\right)
$$

$\hat{\phi}_{i}$ denoting the Fourier transform of $\phi_{i}$. If furthermore $X_{0}$ is countable, the net $\left(\phi_{i}\right)$ may be replaced by a denumerable sequence $\left(\phi_{i}\right)_{i=1}^{\infty}$.

Note. On taking $X_{0}=X$, Theorem (1.6) provides a characterization of the continuous, uniformly almost periodic functions $f$ on $G$.

(1.7) THEOREM. Let $G, X, f$ and $\Phi$ be as in Theorem (1.6). In order that $f$ be the uniform limit on $G$ of Fourier-Stieltjes transforms of elements of $M(X)$, it is necessary and sufficient that

$$
\lim _{i} \int_{G} f \phi_{i} d x=0
$$


for each $L^{1}(G)$-bounded net $\left(\phi_{i}\right)$ extracted from $\Phi$ and such that

for each $m \in M(X)$.

$$
\lim _{i} \int_{X} \hat{\phi}_{i} d m=0
$$

Note. Theorem (1.7) may be compared with a result found jointly by Beurling and Hewitt and stated on p. 138 of Hewitt's survey article [4]. Professor Hewitt informs me that, contrary to what is indicated in the bibliography to [4], the result has not in fact been published to date.

When $G=R$ (the real line) or $Z$ (the discrete group of integers), it is not difficult to display explicitly functions in $B U C(G)$ which are not uniform limits of FourierStieltjes transforms; see, for example, [1, pp. 196-197].

\section{Uniform spans of translates.}

(2.1). Throughout this section, $G$ will denote a Hausdorff locally compact Abelian group and $X$ its character group. Their respective Haar measures, denoted by $d x$ and $d \xi$, are assumed to be adjusted so that the Fourier inversion formula holds without external numerical factors.

We shall employ Theorem (1.5) to prove some results about $T^{*}[f]$, the closure in $B(G)$ (or, what is the same thing, in $B C(G)$ ) of the set of linear combinations of translates of functions $f \in B C(G)$.

(2.2) Spectral notions and generalized Fourier transforms. We review rapidly the concepts used in the main theorems.

(i) The spectrum of a bounded function. We use the definition of the spectrum $\Lambda\left(f_{i}\right)$ given by Herz [1, p. 186]: for $f \in B C(G), \Lambda(f)$ is the set of common zeros of the Fourier-Stieltjes transforms of measures $\mu \in M(G)$ satisfying $\mu * f=0$. Evidently, it comes to the same thing to define $\Lambda(f)$ as the set of common zeros of Fourier transforms of functions $\phi \in L^{1}(G)$ satisfying $\phi * f=0$; and in this form the definition is obviously applicable to any $f \in L^{\infty}(G)$. Moreover, the definition remains still unaltered if $\phi$ is restricted to any dense ideal in $L^{1}(G)$, in particular to the ideal $\Phi=\Phi(G)$ formed of those $\phi \in L^{1}(G)$ for which $\hat{\phi}$ is compact. (Whatever the function $u$ on $X, \boldsymbol{u}$ denotes the support of $u$.)

(ii) Pseudomeasures on $X$. Write, as usual, $A(X)$ for the set of functions $u \in C_{0}(X)$ of the form

$$
u(\xi)=\hat{v}(\xi)=\int_{G} v(x) \overline{\xi(x)} d x
$$

for some $v \in L^{1}(G)$. Then $A(X)$ is a Banach algebra (without unit, if $X$ is noncompact) under the norm $\|u\|_{A}=\|v\|_{1}$. Notice that

$$
A(X) \cap C_{c}(X)=\{\hat{\phi}: \phi \in \Phi(G)\},
$$

and that $A(X)$ is stable under multiplication by Fourier-Stieltjes transforms of elements of $M(G)$. 
By a pseudomeasure on $X$ is meant a continuous linear functional on $A(X)$ (see [12], [13], [15], [16]). These objects form a Banach space $P(X)$ under the dual norm

$$
\|\sigma\|_{A^{\prime}}=\operatorname{Sup}\left\{|\sigma(u)|: u \in A,\|u\|_{A} \leqq 1\right\} .
$$

$P(X)$ is stable under multiplication by Fourier-Stieltjes transforms of elements of $M(G)$.

Given an open subset $U$ of $X$, a pseudomeasure $\sigma \in P(X)$, and a (Radon) measure $\mu$ on $U$, the relation $\sigma=\mu$ on $U$ signifies that

$$
\sigma(u)=\int_{U} u d \mu
$$

for each $u \in A(X) \cap C_{c}(X)$ satisfying $u \subset U$; or, what is equivalent, that

$$
\sigma(\hat{\phi})=\int_{U} \hat{\phi} d \mu
$$

for each $\phi \in \Phi(G)$ satisfying $\hat{\phi} \subset U$. In particular, there exists a maximal open set $U \subset X$ such that $\sigma=0$ on $U$; the closed set $X \cap U^{\prime}$ is the support of $\sigma$, denoted by $\sigma$.

If $X=R^{n}$ or $T^{n}$, there is a natural injection of $P(X)$ into $\mathscr{D}^{\prime}(X)$ (the space of Schwartz distributions on $X$ ).

(iii) The Fourier transform on $L^{\infty}(G)$. If $f \in L^{\infty}(G)$ there is a unique pseudomeasure $\hat{f}$ on $X$ satisfying

$$
\hat{f}(u)=\int_{G} f(x) v(-x) d x \quad\left(u=\hat{v}, v \in L^{1}(G)\right)
$$

or, equivalently,

$$
\hat{f}(\hat{\phi})=\int_{G} f(x) \phi(-x) d x=\phi * f(0) \quad(\phi \in \Phi(G)) .
$$

This $\hat{f}$ is the generalized Fourier transform of $f$. It is easily verified that $f \rightarrow \hat{f}$ is a linear isometry of $L^{\infty}(G)$ onto $P(X)$.

If $\lambda \in M(G)$ and $f \in L^{\infty}(G)$, then $(\lambda * f)^{\wedge}=\hat{\lambda} \cdot \hat{f}$.

If $G=R^{n}$, the pseudomeasure $\hat{f}$ agrees with the Schwartz distribution Fourier transform of $f$.

It can be shown that $\Lambda(f)$ is simply the support of $\hat{f}$, so that $\Lambda(f)=\varnothing$ if and only if $f=0$ l.a.e. (the dual form of Wiener's theorem). From this one may derive rapidly the following three basic properties of $\Lambda(f)$ :

$\left(\Lambda_{1}\right)$. If $f \in L^{\infty}(G), K \in L^{1}(G)$, and $\hat{K}=1$ on a neighbourhood of $\Lambda(f)$, then $f=K * f$ l.a.e. (hence everywhere, if $f$ is continuous); consequently i $f \in B C(G)$ and $\Lambda(f)$ is compact, then $f \in B U C(G)$. 
$\left(\Lambda_{2}\right)$. If $f \in L^{\infty}(G)$ and $K \in L^{1}(G)$, then

$$
\Lambda(K * f) \subset \hat{\boldsymbol{K}} \cap \Lambda(f) .
$$

$\left(\Lambda_{3}\right)$. If $f, g \in L^{\infty}(G)$, then $\Lambda(g) \subset \Lambda(f)$ whenever $g$ is the weak limit in $L^{\infty}(G)$ of linear combinations of translates of $f$; this is the case whenever $g$ is the uniform limit of functions $\mu * f$ with $\mu \in M(G)$, and so in particular whenever $g \in T[f] \cup T^{*}[f]$.

These properties are not stated in the strongest possible form. Observe that $\left(\Lambda_{1}\right)$ and $\left(\Lambda_{2}\right)$ also follow Theorems 1.3 and 1.2 respectively of [1].

(iv) Spectrally null sets. Given $f \in L^{\infty}(G)$ and a closed subset $S$ of $X$, we say that $S$ is spectrally null for $f$ if there exists an open set $U \supset S$ and a measure $\mu$ on $U$ such that $\hat{f}=\mu$ on $U$ and $|\mu|^{*}(S)=0$. (By shrinking $U$ a little, one may always suppose that $\mu \in M(X)$.) It is equivalent to demand that to each $\varepsilon>0$ corresponds an open set $U_{\varepsilon} \supset S$ such that

$$
|\phi * f(0)| \leqq \varepsilon \cdot \operatorname{Sup}|\hat{\phi}|
$$

for each $\phi \in L^{1}(G)$ [or $\left.\Phi(G)\right]$ satisfying $\hat{\phi} \subset U_{\varepsilon}$. Again, in the terminology introduced in (3.2) infra, $S$ is spectrally null for $f$ if and only if the singular support of $\hat{f}$ (i.e., the singular spectrum sing $\Lambda(f)$ of $f$ ) does not meet $S$.

A (not necessarily closed) set $P \subset X$ is said to be spectrally null for $f$, if this last is true for each closed set $S \subset P$.

It is not difficult to verify that if $f \in L^{\infty}(G)$ then an open set $U$ is spectrally null for $f$ if and only if $\hat{f}=0$ on $U$; that a set $P$ is spectrally null for $f$ if and only if the same is true for each compact subset of $P$; and that the union of any locally finite family of sets, each spectrally null for $f$, is itself spectrally null for $f$. However (and herein lies the point of the concept), if $G$ is noncompact, there exist closed sets $S$ which are spectrally null for $f$ and yet which meet $\Lambda(f)$. For example, if $f \in L^{\infty}(G) \cap L^{2}(G)$, any locally null subset of $X$ is spectrally null for $f$, even though $\Lambda(f)$ may well coincide with $X$.

It is evident that if $P$ is spectrally null for $f \in L^{\infty}(G)$, then it is spectrally null for any function $\mu * f$ with $\mu \in M(G)$.

(2.3). The content of $(2.2$, iv) and Theorem (2.4) to follow can be further motivated $a$ posteriori in the following way. The spectrum $\Lambda(f)$, although determining completely those characters $\xi \in X$ which are strict limits of linear combinations of translates of $f$ (see [1, Theorem 1.2 and $(3.6, \mathrm{i})$ infra]), fails to determine which characters are uniform limits of linear combinations of translates of $f$. For example, if $u \in C_{c}(G)$ and $u \neq 0$, consider the functions $f_{1}=1+u$ and $f_{2}=u$. Then, if $G=R$ (the real line), the remarks in $(2.2$, iii) show that the spectra of $f_{1}$ and of $f_{2}$ are both the whole of $X$ (here identifiable with $R$ ). On the other hand, it follows from Corollary (2.5) that $1 \in T\left[f_{1}\right]$; and yet evidently $T\left[f_{2}\right] \subset C_{0}(G)$ and so $1 \notin T\left[f_{2}\right]$. (In the second clause of $(2.5)$ we take $f=f_{1}=1+u$ and $c=1$ and apply the remarks in $(2.2, \mathrm{iii})$.) 
However, Theorem (2.4) constitutes a partial substitute as a comparison theorem, implying as it does (via Corollary (2.5)) that $\xi \in T^{*}\left[f_{1}\right]$ and $\xi \in T^{*}\left[f_{2}\right]$ are equivalent assertions, whenever there is a neighbourhood of $\xi$ which is spectrally null for $f_{1}-f_{2}$.

We now proceed to the statement and proof of Theorem (2.4).

(2.4) THeOREM. Suppose that $f, f_{0}, g \in B C(G)$, that $g \in T^{*}\left[f_{0}\right]$, and that there exists a neighbourhood $U$ of $\Lambda(g)$ for which $\Lambda\left(f_{0}\right) \cap U$ is spectrally null for $f-f_{0}$. Then $T^{*}[g] \subset T^{*}[f]$, and in particular $g \in T^{*}[f]$ if $g$ is uniformly continuous.

Proof. We break this into three stages.

(1) The general result will follow as soon as it is established that $g \in T^{*}[f]$ if $\Lambda(g)$ is compact, the other hypotheses remaining fixed. For if $g$ satisfies these hypotheses, so too does $K * g$ for any $K \in \Phi$, by virtue of $\left(\Lambda_{2}\right)$. The supposedly established special case then entails that $K * g \in T^{*}[f]$ for any $K \in \Phi$. Hence, since $\Phi$ is dense in $L^{1}(G)$, the same is true for any $K \in L^{1}(G)$, and consequently $T^{*}[g] \subset T^{*}[f]$.

(2) Suppose then that $\Lambda(g)$ is compact and the other hypotheses are maintained. Choose a compact neighbourhood $N$ of $\Lambda(g), N \subset U$, and then a function $k \in L^{1}(G)$ such that $\hat{k}=1$ on some neighbourhood of $\Lambda(g)$ and $\hat{k} \subset N$. By $\left(\Lambda_{1}\right)$, $g=k * g$, which belongs to $T^{*}\left[k * f_{0}\right]=T^{*}\left[f_{0}^{\prime}\right]$, say. By $\left(\Lambda_{2}\right)$,

$$
\Lambda\left(f_{0}^{\prime}\right) \subset \hat{\boldsymbol{k}} \cap \Lambda\left(f_{0}\right) \subset N \cap \Lambda\left(f_{0}\right) .
$$

Besides this, $\left(\Lambda_{3}\right)$ shows that $\Lambda(g) \subset \Lambda\left(f_{0}\right)$, and so, by $\left(\Lambda_{2}\right)$ again, that

$$
\Lambda(g)=\Lambda(k * g) \subset \hat{\boldsymbol{k}} \cap \Lambda(g) \subset N \cap \Lambda\left(f_{0}\right) .
$$

Also, $N \cap \Lambda\left(f_{0}\right) \subset U \cap \Lambda\left(f_{0}\right)$ is spectrally null for $f-f_{0}$ and therefore for $f^{\prime}-f_{0}^{\prime}$, if we write $f^{\prime}=k * f$.

Putting $S=N \cap \Lambda\left(f_{0}\right)$, we see that $S$ is compact, and we have just verified that $g \in T^{*}\left[f_{0}^{\prime}\right]$, that $\Lambda(g) \subset S$, that $\Lambda\left(f_{0}^{\prime}\right) \subset S$, and that $S$ is spectrally null for $f^{\prime}-f_{0}^{\prime}$. If we can deduce from this that $g \in T^{*}\left[f^{\prime}\right]$, then a fortiori we shall have $g \in T^{*}[f]$, and the proof will be complete.

(3) We are thus reduced to proving the following: if $f, f_{0}, g \in B C(G)$ and $S$ is a compact subset of $X$, and if

(i) $\Lambda(g) \subset S$,

(ii) $\Lambda\left(f_{0}\right) \subset S$,

(iii) $S$ is spectrally null for $f-f_{0}$, and

(iv) $g \in T^{*}\left[f_{0}\right]$,

then $g \in T^{*}[f]$.

To do this, we write $f=f_{0}+f_{1}$. By (iii), to each $\varepsilon>0$ corresponds a neighbourhood $U_{\varepsilon}$ of $S$ such that 


$$
\left|\phi * f_{1}(0)\right| \leqq \varepsilon \cdot \operatorname{Sup}|\hat{\phi}|
$$

for each $\phi \in L^{1}(G)$ satisfying $\hat{\phi} \subset U_{e}$. Next choose for each such $\varepsilon$ a function $k_{\varepsilon} \in L^{1}(G)$ so that $k_{\varepsilon}=1$ on a neighbourhood of $S, 0 \leqq k_{z} \leqq 1$, and $\hat{k}_{z} \subset U_{z}$ [5, p. 146]. According to $\left(\Lambda_{1}\right)$, (i), and (ii), $f_{0}=k_{\varepsilon} * f_{0}$ and $g=k_{\varepsilon} * g$. Put $f_{\varepsilon}^{\prime}=k_{\varepsilon} * f$.

We shall use Theorem (1.5) and consider any net $\left(\phi_{i}\right)$ in $L^{1}(G)$ satisfying

$$
M=\operatorname{Sup}_{i} \int_{G}\left|\phi_{i}\right| d x<\infty
$$

and

$$
\lim _{i} \phi_{i} * K * f(0)=0
$$

for each $K \in L^{1}(G)$. Then, since $\left|f_{\varepsilon}^{\prime} \in\right| T^{*}[f]$, it follows that also

$$
\lim _{i} \phi_{i} * K * \mid \mathrm{fl}_{e}^{\prime}(0)=0
$$

for each $K \in L^{1}(G)$. Since $k_{\varepsilon} \times f_{0}=f_{0}$, we have for any $K \in L^{1}(G)$

$$
\phi_{i} * K * f_{\varepsilon}^{\prime}=\phi_{i} * K * \mid f_{0}+\phi_{i} * k_{\varepsilon} * K * I f_{1}
$$

and therefore

$$
\left|\phi_{i} * K *\right| f_{0}(0)|\leqq| \phi_{i} * K * f_{\varepsilon}^{\prime}(0)|+| \phi_{i} * K * k_{\varepsilon} * f_{1}(0) \mid .
$$

Applying (2.4.1), with $\phi$ replaced by $\phi_{i} * k_{\varepsilon} * K$, and using (2.4.5), it follows that

$$
\left|\phi_{i} * K * f_{0}(0)\right| \leqq\left|\phi_{i} * K * f_{e}^{\prime}(0)\right|+M \varepsilon \quad \text { Sup }|R| .
$$

This combines with (2.4.4) to yield

$$
\limsup _{\|}\left|\phi_{i} * K * f_{0}(0)\right| \leqq M \varepsilon \quad \operatorname{Sup}|R| .
$$

Since $\varepsilon>0$ is arbitrary, we infer that

$$
\lim _{i} \phi_{i} * K * f_{0}(0)=0
$$

for each $K \in L^{1}(G)$. In view of (iv) and (2.4.2), this last entails that

$$
\lim _{i} \phi_{i} * g(0)=0 \text {. }
$$

Thus, for any net $\left(\phi_{i}\right)$ in $L^{1}(G),(2.4 .2)$ and (2.4.3) together imply (2.4.6). Theorem (1.5) then shows that indeed $g \in T^{*}[f]$, as we wished to prove. 
(2.5) Corollary. Suppose that $f, f_{0} \in B C(G)$, that $\xi \in X$ satisfies $\xi \in T^{*}\left[f_{0}\right]$, and that there is a neighbourhood $U$ of $\xi$ such that $\Lambda\left(f_{0}\right) \cap U$ is spectrally null for $f-f_{0}$. Then $\xi \in T^{*}[f]$. In particular, $\xi \in T^{*}[f]$ provided $f \in B C(G)$ is such that, for some number $c \neq 0,\{\xi\}$ is spectrally null for $f-c \xi$.

Proof. This is immediate from Theorem (2.4) on taking $g=\xi$, so that $\Lambda(g)=\{\xi\}$. For the second clause we specialize still more by taking $f_{0}=c \xi$, for which $\Lambda\left(f_{0}\right)=\{\xi\}$.

(2.6) REMARKS.

(i) Detailed examples of "comparison functions" $f_{0}$ for use in (2.4) and (2.5) appear in (2.9) infra.

(ii) If $f \in B C(G), \xi \in X$, and $\hat{f}$ is equal to a measure $\mu$ on a neighbourhood of $\xi$ such that $\mu(\{\xi\}) \neq 0,(2.5)$ shows that $\xi \in T^{*}[f]$. It is not difficult to show that, conversely, $\mu(\{\xi\}) \neq 0$ whenever $\xi \in T^{*}[f]$.

(iii) Both (2.4) and (2.5) have analogues for spans of translates, the proofs being based upon (1.5A) in place of (1.5). For example: suppose that $f_{0} \in B C(G)$, that $f, g \in B U C(G)$, that $g \in T\left[f_{0}\right]$, and that there exists a neighbourhood $U$ of $\Lambda(g)$ such that $\Lambda\left(f_{0}\right) \cap U$ is spectrally null for $f-f_{0}$; then $g \in T[f]$.

(iv) Whilst (2.4) yields useful information about $T^{*}[f]$ whenever a suitable comparison function $f_{0}$ is available, the next theorem aims at information about $T^{*}[f]$ without the intervention of a comparison function.

(2.7) TheOREM. (i) Suppose that $f \in B C(G)$ and that $\hat{f}$ is a measure $\mu$. Then $T^{*}[f]$ contains every $g \in B C(G)$ such that $\hat{g}$ is a bounded measure $v$ which is absolutely continuous relative to $\mu$, i.e., every $g$ which is of the form

$$
g(x)=\int_{X} \xi(x) s(\xi) d \mu(\xi),
$$

where $s$ is $\mu$-integrable.

(ii) If $f \in B C(G)$ is such that $\hat{f}$ is a function $F \neq 0$ l.a.e., then $T^{*}[f] \supset C_{0}(G)$.

Proof. (i) By Theorem (1.5) it suffices to show that if $\left(\phi_{i}\right)$ is an $L^{1}(G)$-bounded net extracted from $\Phi$, and if

$$
\lim _{i} \phi_{i} * K * f(0)=0 \quad\left(K \in L^{1}(G)\right)
$$

then

$$
\lim _{i} \phi_{i} * g(0)=0
$$

for each $g$ of the stated type.

Now, (2.7.1) implies that

$\lim \phi * \phi_{i} * f(0)=0$ 
for each $\phi \in \Phi$. Using the fact that $\hat{f}$ is the measure $\mu$, this last equation may.be rewritten

$$
\lim _{i} \int_{X} \hat{\phi}(\xi) \hat{\phi}_{i}(\xi) d \mu(\xi)=0
$$

Consider next the function $s$ figuring in the definition of $g$. We claim that, given $\varepsilon>0$, we can choose $\phi \in \Phi$ so that

$$
\int_{X}|s-\hat{\phi}| d \mu \leqq \varepsilon .
$$

Indeed, if $h \in L^{\infty}(X, \mu)$ satisfies $\int_{X} \hat{\phi} h d \mu=0$ for all $\phi \in \Phi$, then also (since $\Phi$ is an algebra)

$$
\int_{X} \hat{\phi} \hat{\psi} h d \mu=0
$$

for all $\phi, \psi \in \Phi$. As is well known, the $\hat{\psi}$ with $\psi \in L^{1}(G)$ are uniformly dense in $C_{0}(X)$; the same is therefore true even if $\psi$ be restricted to $\Phi$. The last-written equation therefore entails that $\hat{\phi} h=0$ l.a.e. $(\mu)$ for each $\phi \in \Phi$; and this in turn shows that $h=0$ l.a.e. $(\mu)$. The Hahn-Banach theorem now affirms the possibility of the approximation (2.7.4).

Since the transforms $\hat{\phi}_{i}$ are uniformly bounded, (2.7.3) and (2.7.4) combine to show that

$$
\lim \sup _{i}\left|\int_{X} s \hat{\phi}_{i} d \mu\right| \leqq \varepsilon .
$$

This being the case for any $\varepsilon>0$, we must conclude that

$$
\lim _{i} \int_{X} s \hat{\phi}_{i} d \mu=0
$$

Since $\hat{g}$ is the measure $v=s \cdot \mu,(2.7 .5)$ signifies that

which is (2.7.2). This proves (i).

$$
\lim _{i} \phi_{i} * g(0)=0
$$

(ii) In this case we can make use of part of the preceding argument, taking $d \mu(\xi)=F(\xi) d \xi$. Equation (2.7.3) itself yields the conclusion that $T^{*}\left[i f_{i}\right]$ will contain any function $g$ of the form

$$
g(x)=\int_{X} \xi(x) \hat{\phi}(\xi) F(\xi) d \xi
$$

where $\phi \in \Phi$. It suffices therefore to show that the functions $\hat{\phi} F$ are dense in $L^{1}(X)$. But this can be done in much the same way as the approximation (2.7.4) was shown to be possible, bearing in mind now that $F$ is nonvanishing la.e. This will complete the proof of (ii). 
Remark. For $G=R^{m}$ and $f \in C_{0}(G)$, a similar result appears as Theorem 4 in Edwards [6]. See also (3.4) and (3.5) infra.

(2.8) REMARKS.

(i) It is possible to give necessary and sufficient conditions on $f$ in order that $\hat{f}$ shall be a measure on a given open subset $U$ of $X$. One such criterion is the existence of a net $\left(f_{i}\right)$ of functions in $L^{\infty}(G) \cap L^{1}(G)$ converging weakly in $L^{\infty}(G)$ to $f$ and such that

$$
\lim \sup _{i} \int_{C}\left|\hat{f}_{i}\right| d \xi<\infty
$$

for each compact set $C \subset U$.

Another such criterion is that $f$ be the weak limit in $L^{\infty}(G)$ of the FourierStieltjes transforms of a net $\left(\mu_{i}\right)$ of bounded Radon measures on $X$ such that

$$
\lim \sup _{i} \int_{C} d\left|\mu_{i}\right|<\infty
$$

for each compact set $C \subset U$.

The property in question is a purely local one. More precisely, in order that $\hat{f}$ be equal to a measure on $U$, it is necessary and sufficient that to each $\xi \in U$ corresponds an open neighbourhood $U_{\xi}$ of $\xi$, and to each compact set $C \subset U_{\xi}$ a number $m(C)<\infty$, such that

$$
\mid \phi * f(0|\leqq m(C) \cdot \operatorname{Sup}| \hat{\phi} \mid
$$

for each $\phi \in L^{1}(G)[$ or $\Phi(G)]$ satisfying $\hat{\phi} \subset C$.

(ii) Using an obvious notation, if $f \in L^{\infty}\left(G_{k}\right)(k=1,2)$ is such that $\hat{f}_{k}$ is a measure $\mu_{k}$ on the open set $U_{k} \subset X_{k}$, then the function $f$ on $G_{1} \times G_{2}$ defined by

$$
f\left(x_{1}, x_{2}\right)=f_{1}\left(x_{1}\right) f_{2}\left(x_{2}\right)
$$

has a transform $\hat{f}$ which is equal on $U_{1} \times U_{2}$ to the measure $\mu_{1} \otimes \mu_{2}$. The verification is immediate.

(2.9) SOME EXAMPLES. Specific applications of Theorem (2.4) and Corollary (2.5) depend upon a supply of comparison functions $f_{0}$ for which enough is known about $T^{*}\left[f_{0}\right]$.

One source of such comparison functions is Theorem (2.7) in conjunction with the remarks in (2.8); it is especially useful in connection with functions given as Fourier-Stieltjes transforms or as suitable limits of these.

A second source comprises the set of uniformly almost periodic (u.a.p.) functions on $G$. If $u$ is u.a.p., $T^{*}[u]=T[u]$ can in theory be completely determined in the following way. Imbed $G$ topologically and algebraically as a dense subgroup of its Bohr compactification $b G$ and use the well-known fact that u.a.p. functions on $G$ are precisely the restrictions to $G$ of the continuous functions on $b G$. Since $b G$ is compact Abelian, if $h$ is a continuous function on $b G, T[h]=T^{*}[h]$ 
consists exactly of those continuous functions $k$ on $b G$ such that $\hat{k}$ vanishes at all points of $X$ at which $\hat{h}$ vanishes. Interpreted in terms of u.a.p. functions on $G$, this means that if we introduce the Bohr spectrum

$$
\lambda(u)=\{\xi \in X:(\text { mean value of } u \bar{\xi}) \neq 0\}
$$

of the u.a.p. function $u$, then $T[u]=T^{*}[u]$ contains precisely those u.a.p functions $v$ such that $\lambda(v) \subset \lambda(u)$. It is also well known (and easily deducible from the theory for the compact group $b G$ ) that any u.a.p. function $u$ is the uniform limit of linear combinations of characters $\xi \in \lambda(u)$, these last being just the characters belonging to $T[u]$. On the other hand, it is not difficult to verify that $\Lambda(u)=\overline{\lambda(u) \text {; }}$ accordingly, if $\lambda(u)$ is not closed, there are characters $\xi \in \Lambda(u)$ which do not belong to $T[u]$.

If $u$ is actually periodic, with a period subgroup $P$ such that $G / P$ is compact, the situation is very simple. For then the annihilator $P^{0}$ of $P$ in $X$, being isomorphic as a topological group with the character group of $G / P$, is discrete. The transform of $u$ is the measure

$$
\hat{u}=\sum_{\xi \in P^{0}} c(\xi) \varepsilon_{\xi},
$$

the series converging vaguely, where

$$
c(\xi)=\int_{G / P} u(\bar{x}) \xi(\bar{x}) d \bar{x}
$$

and $u(\bar{x})=u(x)$ for $x \in \bar{x} \in G / P$. So $\Lambda(u)=\lambda(u)$ is now the set of $\xi \in P^{0}$ for which $c(\xi) \neq 0$.

We shall now consider a few examples of a much more special nature, most of which are not covered by the preceding remarks. When speaking of a group $G=R^{n}$ we shall usually identify $X$ with $R^{n}$ in the usual fashion, identifying thereby a point $\xi$ of $R^{n}$ with the corresponding character $x \rightarrow \exp (2 \pi i \xi \cdot x)$. The choice of the factor $2 \pi$ arranges that the adjusted Haar measures are then numerically identical with the Lebesgue measure on $R^{n}$. Free use will be made of the remarks in $\left(2.2\right.$, iii). Notice that examples for $G=R^{n}$ may be derived from those for $G=R$ by use of $(2.8$, ii).

(i) For $G=R$ the classical theory of Fourier integrals provides a number of criteria ensuring that a (not necessarily integrable) function $f$ has a transform which is a function; for this, see [8, pp. 169-174]. Such criteria normally apply only to functions in $C_{0}(R)$. There are, incidentally, analogous results for the case $G=Z$ (the discrete additive group of integers); see [7, Volume I, p. 185]. A different type of example for $G=R$ stems from a result of Wiener given in [7, Volume II, p. 298]. This shows in particular that if $f \in L^{\infty}(R)$ is such that

$$
\sum_{n=-\infty}^{\infty}\left(\int_{n}^{n+1}|f(x)| d x\right)^{2}<\infty
$$


then $f$ is a function satisfying

$$
(1+|\xi|)]^{-1} \hat{f}(\xi) \in L^{2}(R)
$$

(ii) Take again $G=R$. From [9, Tome II, p. 126] we read off the pair of transforms

$$
f(x)=\exp \left(i \pi x^{2}\right), \hat{f}(\xi)=2^{-1 / 2}(1+i) \exp \left(-i \pi \xi^{2}\right) .
$$

Consequently for real $a \neq 0$ we may derive the pair of transforms

$$
f(x)=\exp \left(i \pi a x^{2}\right), \quad \hat{f}(\xi)=C_{a} \cdot \exp \left(-i \pi \xi^{2} / a\right),
$$

where $C_{a} \neq 0$ depends upon $a$ only.

More generally, consider $f(x)=\exp [i P(x)]$ where $P$ is a nonlinear polynomial with real coefficients. Applying the Fourier-Schwartz transformation to the equation $d f / d x=i P^{\prime}(x) f$, one derives the relation $2 \pi i \xi \hat{f}=i P^{\prime}\left[(-2 \pi i)^{-1} d / d \xi\right] \hat{f}$. Reference to [9, Tome I, p. 129] confirms that this differential equation has only the usual solutions, which are all analytic. It follows that $f(x)=\exp [i P(x)]$ satisfies the conditions in Theorem $(2.7, \mathrm{ii})$, so that we may assert that to each $g \in C_{0}(R)$ corresponds a sequence of functions $\left(K_{n}\right)_{n=1}^{\infty}$ extracted from $L^{1}(R)$ such that

$$
\lim _{n} \int_{-\infty}^{\infty} K_{n}\left(x^{\prime}\right) \exp \left[i P\left(x-x^{\prime}\right)\right] d x^{\prime}=g(x)
$$

uniformly for $x \in R$.

Again, if $\boldsymbol{P}$ is a nonconstant polynomial with real coefficients, and if $f(x)=\sin ^{2} P(x)$, it is easily seen that

$$
\hat{f}(\xi)=\frac{1}{2} \varepsilon+\lambda,
$$

where $\lambda$ is either a function (if $P$ is nonlinear) or a measure whose support does not contain 0 (if $P$ is linear and nonconstant). Corollary (2.5) is applicable if we take $f_{0}(x)=\frac{1}{2}, g(x)=1$; for the transform of $f-f_{0}$ is the measure $\lambda$, and $\Lambda\left(f_{0}\right)=\{0\}$ is spectrally null for $f-f_{0}$. Since, obviously, $g \in T^{*}\left[f_{0}\right]$, it follows that $g=1 \in T^{*}[f]$. In other words, there exists a sequence $\left(K_{n}\right)_{n=1}^{\infty}$ of integrable functions such that

$$
\lim _{n} \int_{-\infty}^{\infty} K_{n}\left(x^{\prime}\right) \sin ^{2} l P\left(x-x^{\prime}\right) d x^{\prime}=1
$$

uniformly for $x \in R$. The same conclusion is valid if $\sin ^{2} P(x)$ is replaced by any linear combination $A \sin ^{2} P(x)+B \cos ^{2} P(x)$, where $A$ and $B$ are complex and $A:+B \neq 0$. 
Similarly, if $G=R^{n}$, if $a$ is real and nonzero, and if

$$
f(x)=A \sin ^{2}\left(i \pi a r^{2}\right)+B \cos ^{2}\left(i \pi a r^{2}\right),
$$

where $r=|x|$ (the Euclidean length of $x \in R^{n}$ ) and $A+B \neq 0$, then $1 \in_{i}^{*}\left[f_{i}\right]$.

(iii) Further interesting examples for $G=R^{n}$ (with emphasis on the cases where $n>1$ ) are obtainable in the shape of functions of the form

$$
f(x)=A(x) B(x) / Q(x) \not \equiv 0,
$$

assumed to belong to $B C\left(R^{n}\right)$, where

(a) $\hat{A}$ has a compact support,

(b) $\hat{B}$ is an analytic function on $R^{n}$, and

(c) $Q(x)$ is an elliptic polynomial.

Here (c) means that the homogeneous part of $Q$ of highest degree is nonvanishing for $x \neq 0$ in $R^{n}$, so that the associated differential operator $D=Q\left[(-2 \pi i)^{-1} \partial / \partial \xi\right]$ is elliptic [10, pp. 177-178].

The transform $\hat{f}$ satisfies the differential equation

$$
D \hat{f}=\hat{A} * \hat{B},
$$

the right-hand side of which is analytic on $R^{n}$. The ellipticity of $D$ shows that $\hat{f}$ also is analytic on $R^{n}$. Since $f \not \equiv 0$, the zeros of $\hat{f}$ form a null set. (If $n=11$, this is entirely elementary; if $n>1$, the question may be settled by noting its local nature and then taking into account the local expression of $\hat{f}$ afforded by the Weierstrass Vorbereitungssatz.)

From $\left(2.7\right.$, ii) we conclude therefore that $T^{*}[f] \supset C_{0}\left(R^{n}\right)$; and from (2.4) it follows that if $f_{0} \in B C\left(R^{n}\right), \mathrm{g} \in B U C\left(R^{n}\right)$, and $\Lambda\left(f_{0}\right)$ is null, then the inclusion $g \in T^{*}\left[f_{0}\right]$ implies that also $g \in T^{*}\left[f_{0}+f\right]$.

Were we to take in place of (2.9.4) a function of the form

$$
f(x)=A(x) / Q(x) \not \equiv 0
$$

with $A$ and $Q$ as before, the situation is less simple. For $\hat{f}$ one would now have the equation

$$
D \hat{f}=\hat{A},
$$

and the smoothness properties of $\hat{f}$ depend almost entirely on those of $\hat{A}$ and:of any one elementary solution $E$ for $D$. Thus, it is certain that $\hat{f}$ is analytic on $R^{n} \cap K^{\prime}$ (where $K$ is the compact support of $\hat{A}$ ); on the other hand, in the neighbourhood of $K$ we have

$$
\hat{f}=E * A+\alpha,
$$

where $\alpha \in C\left(R^{n}\right)$. (This last is true even if we weaken (c) to the demand that $Q$ be hypoelliptic.) Accordingly, $\hat{f}$ is a function if and only if $E * \hat{A}$ is a function. in 
which case the way is open to apply (2.4). Otherwise a more detailed examination of $E * A$ is required.

As an example we might take

$$
Q(x)=Q_{a, s}(x)=\left(a+4 \pi^{2}|x|^{2}\right)^{s},
$$

where $\operatorname{Re} a>0$ and $s$ is a natural number, so that $D$ is the sth power of the metaharmonic operator $a-\Delta$. An associated elementary solution $E=E_{a, s, n}$ is explicitly known, viz.

where

$$
E_{a, s, n}=(-1)^{s-1}(\partial / \partial a)^{s-1} E_{a, n} /(s-1) !,
$$

$E_{a, n}= \begin{cases}a^{(n-2) / 2}\left[\omega_{n}(n-2) !\right]^{-1} \int_{1}^{\infty} \exp \left[-a^{1 / 2}|\xi| t\right]\left(t^{2}-1\right)^{(n-3) / 2} d t \quad \text { if } n \geqq 2, \\ \frac{1}{2} a^{-1 / 2} \exp \left[-a^{1 / 2}|\xi|\right] \quad \text { if } n=1,\end{cases}$

and $\omega_{n}=2 \pi^{n / 2} / \Gamma\left(\frac{1}{2} n\right) ;$ (see $[11$, pp. $59,60,65]$ ).

(iv) The formula (2.9.1) suggests a fairly general class of examples, as we shall now show.

Take a quadratic form $Q$ on $R^{n}$ of the type

$$
Q(x)=\sum_{r=1}^{n} a_{r} x_{r}^{2},
$$

where the $a_{r}$ are real and nonzero, and put

$$
Q^{\prime}(\xi)=-\pi^{2} \sum_{r=1}^{n} \xi_{r}^{2} / a_{r}
$$

Parallel with (2.9.1) is the one-variable formula

$$
\left[\exp \left(i a x^{2}\right)\right]^{\wedge}=\left(2 \pi^{-1}|a|\right)^{-1 / 2}(1+i \operatorname{sgn} a) \exp \left(-i \pi^{2} \xi^{2} / a\right),
$$

whence follows the formula for $n$ real variables:

$$
[\exp i Q(x)]^{\wedge}=C_{n}(a) \cdot \exp i Q^{\prime}(\xi)
$$

where

$$
C_{n}(a)=\prod_{r=1}^{n}\left(2 \pi^{-1}\left|a_{r}\right|\right)^{-1 / 2}\left(1+i \operatorname{sgn} a_{r}\right) .
$$

Let now $u$ be any continuous function defined on the unit circumference $|z|=1$ of the complex plane, and define $f \in B U C\left(R^{n}\right)$ by $f(x)=u[\exp i Q(x)]$. We claim that

$$
\hat{f}=\gamma_{0} \varepsilon+F,
$$

where $F$ is a continuous function and 


$$
\gamma_{0}=(2 \pi)^{-1} \int_{0}^{2 \pi} u\left(e^{i t}\right) d t .
$$

Indeed, for $u$ one has a development

$$
u(z)=\sum_{N=-\infty}^{\infty} \gamma_{N} z^{N},
$$

where

$$
\gamma_{N}=(2 \pi)^{-1} \int_{0}^{2 \pi} u\left(e^{i t}\right) e^{-i N t} d t
$$

the series (2.9.12) being uniformly summable $(C, 1)$. Consequently,

$$
f(x)=\gamma_{0}+\sum_{N \neq 0} \gamma_{N} \exp i N Q(x),
$$

this series also being uniformly summable. Using (2.9.9), it follows that

where

$$
\hat{f}=\gamma_{0} \varepsilon+\sum_{N \neq 0} \gamma_{N} \cdot C(n, a, N)|N|^{-n / 2} \exp \left[i Q^{\prime}(\xi) / N\right]
$$

$$
C(n, a, N)=\prod_{r=1}^{n}\left(2 \pi^{-1}\left|a_{r}\right|\right)^{-1 / 2}\left(1+i \operatorname{sgn} N a_{r}\right),
$$

the series being summable for the distributional topology. The proof may then be completed by showing that the series

$$
\sum_{N \neq 0} \gamma_{N} \cdot C(n, a, N)|N|^{-n / 2} \exp \left[i Q^{\prime}(\xi) / N\right]
$$

is locally uniformly convergent on $R^{n}$; the details of the argument are omitted. As especially simple examples we may take $u(z)=\frac{1}{2}\left(z+z^{-1}\right)$ or $\frac{1}{2} i^{-1}\left(z+z^{-1}\right)$, in which cases $f(x)=\cos Q(x)$ or $\sin Q(x)$ and $\gamma_{0}=0$, so that $f$ is a continuous function. Likewise, if $u(z)=\frac{1}{2}\left|z \pm z^{-1}\right|$, then $f(x)$ is $|\cos Q(x)|$ or $\mid \sin 6(x) \eta$, and $\hat{f}=\gamma_{0} \varepsilon+F$ with $\gamma_{0}>0$ and $F$ a continuous function.

(v) Returning to the case of a general (Hausdorff locally compact Abelian) $G$, we record one fairly general derivative of Theorem (2.4).

Suppose that $f_{0}, g \in B C(G)$ and the disjoint closed subsets $S, F$ of $X$ are such that

(a) $\Lambda(g) \subset S$;

(b) $\Lambda\left(f_{0}\right) \subset S \cup F$;

(c) $g \in T^{*}\left[f_{0}\right]$.

Suppose further that

$$
f(x)=f_{0}(x)+\sum_{n=1}^{\infty} f_{n}(x)
$$

belongs to $B C(G)$, where $f_{n} \in L^{\infty}(G)$ and the series converges weakly in $L^{\infty}(G)$. 
Suppose finally that there exists an open set $U \supset S$ and measures $\mu_{n}(n=1,2, \cdots)$ on $U$ such that $\hat{f}_{n}=\mu_{n}$ on $U$ and

(d) the support $P_{n}$ of $\mu_{n}$ is such that $P_{n} \rightarrow \infty$ in $U$ as $n \rightarrow \infty$ (i.e., each compact subset of $U$ meets $P_{n}$ for at most finitely many $n$ );

(e) $\left|\mu_{n}\right|(S)=0$ for $n=1,2, \cdots$.

Then $T^{*}[g] \subset T^{*}[f]$; and $g \in T^{*}[f]$ if $g$ is uniformly continuous.

Proof. Condition (d) ensures that the series $\sum_{n=1}^{\infty} \mu_{n}$ converges vaguely on $U$ to a measure $\mu$ on $U$. In conjunction with (e), (d) shows also that $|\mu|(C)=0$ for each compact set $C \subset S$, whence it follows that

$$
|\mu|(S)=0 \text {. }
$$

The vague convergence of this same series of measures, together with the assumed weak convergence of the series appearing in (2.9.15), entails at once that the transform of $f-f_{0}$ is equal on $U$ to $\mu$. Hence, by (2.9.16), $S$ is spectrally null for $f-f_{0}$.

The result now follows at once from Theorem (2.4), on taking $U=X \cap F^{\prime}$.

An appeal to Corollary (2.5) leads similarly to the following result:

(vi) Suppose that $\xi \in X$, that $c \neq 0$ is a constant, and that

$$
f(x):=c \xi(x)+\sum_{n=1}^{\infty} f_{n}(x),
$$

where $f_{n} \in L^{\infty}(G)$, where the series converges weakly in $L^{\infty}(G)$, and where $f \in B C(G)$. Suppose also that there is a neighbourhood $U$ of $\xi$ and measures $\mu_{n}$ on $U$ such that $\hat{f}_{n}=\mu_{n}$ on $U$, the $\mu_{n}$ satisfying (d) of $(2.9$, vii) and also

$$
\mu_{n}(\{\xi\})=0 \quad(n=1,2, \cdots) .
$$

Then $\xi \in T^{*}[f]$.

Proof. To the proof of $(2.9, v)$ it is necessary only to add the observation that the relation $\mu_{n}(\{\xi\})=0$ is equivalent to $\left|\mu_{n}\right|(\{\xi\})=0$.

Note. If $G=R$ and $\xi(x)=1$, the conditions are satisfied if $f_{n}(x)=c_{n}[u(x)]^{n}$, where $u \in L^{\infty}(G), \hat{u}=\mu$ globally, $\mu(\{0\})=0$, all convolution powers of $\mu$ exist absolutely and the support $P$ of $\mu$ is such that

$$
P_{n}=P+\cdots+P(n \text { summands }) \rightarrow \infty
$$

as $\boldsymbol{n} \rightarrow \infty$. Notice that since $R$ has no nonzero idempotents (for its additive group structure), each convolution power $\pi$ of $\mu$ satisfies $\pi(\{0\})=0$ whenever $\mu(\{0\})=0$. Moreover (2.9.18) is satisfied whenever the support $P$ lies in a half-line $\xi \geqq p$, or a half-line $\xi \leqq-p$, where $p>0$.

(2.10) An APplication. The relationship between Wiener's Tauberian theorem and his "closure of translations" theorem suggests the following simple observation. 
If $\mu \in M(G)$ and $f \in B C(G)$, then the relations

$$
\mu * f \in C_{0}(G)
$$

and

(2.10.2) $g$ is the uniform limit of functions $\lambda * f$ with $\lambda \in M(G)$

together entail that

$$
\mu * g \in C_{0}(G) \text {. }
$$

Moreover, if $\mu$ is replaced by a function $K \in L^{1}(G)$, it is simple to formulate a corresponding assertion for functions $f$ and $g$ in $L^{\infty}(G)$. Also, if $G=R$, the limits at infinity implied in (2.10.1) and (2.10.3) could be replaced by one-sided limits (as $x \rightarrow+\infty$ or as $x \rightarrow-\infty$ in place of $|x| \rightarrow \infty$ ).

Consequently, given (2.10.1) and the relation

$$
1 \in T^{*}[f] \cup T[f],
$$

there follows the conclusion

$$
\int_{G} d \mu=0
$$

For example, if $G=R$ and $P$ is any nonconstant polynomial with real coefficients, then the relation

$$
\lim _{x \rightarrow \infty} \int_{R} \sin ^{2} P\left(x-x^{\prime}\right) d \mu\left(x^{\prime}\right)=0
$$

entails

$$
\int_{R} d \mu(x)=0
$$

(We are here using the result obtained in $(2.9$, ii).)

In particular, the only positive bounded Radon measure $\mu$ on $R$ satisfying (2.10.6) is $\mu=0$.

3. Subspaces of $B C(G)$ and admissible topologies. In this section we shall consider briefly certain translation-invariant vector subspaces $V$ of $B C(G)$ and admissible topologies on them. In this way one may in particular recover some results about approximation in the strict topology.

(3.1) Admissible pairs. For brevity we shall term "admissible pair" an object $(V, \theta)$, where $V$ is is a translation-invariant vector subspace of $B C(G)$ and $\theta$ is a locally convex vector space topology on $V$ such that each $\theta$-continuous linear functional $F$ on $V$ is representable in the form 


$$
F(f)=\int_{G} f(-x) d \mu(x)
$$

for all $f \in V, \mu$ being a suitably chosen element of $M(G)$.

In principle, Mackey's theorem (see [2, Theorem 8.3.2]) permits a description of all such admissible pairs, granted the identification of all convex and $\sigma(M(G), V)$-compact subsets of $M(G)$. We are not here concerned with any such description. Instead we merely note two concrete and particularly significant examples.

(i) $V=C_{0}(G)$ and $\theta$ is the topology of uniform convergence. That one has here an admissible pair, is the content of the Riesz representation theorem, or of the Bourbaki definition of Radon measures, according to one's point of view.

(ii) $V=B C(G), \theta$ is the strict topology defined by the seminorms

$$
N_{k}(f)=\|k f\|,
$$

$k$ ranging over $C_{0}(G)$. We omit the proof of admissibility.

Suppose now that $(V, \theta)$ is an admissible pair. For $f \in V$, denote by $T_{\theta}[f]$ the $\theta$-closed vector subspace of $V$ generated by the translates of $f$. (There is no point in considering separately, in those cases in which $V$ is a convolution module over $L^{1}(G)$, the $\theta$-closure $T_{\theta}^{*}[f]$ in $V$ of $L^{1}(G) * f$.) According to the Hahn-Banach theorem, given $f$ and $g$ in $V$, one has $g \in T_{\theta}[f]$ if and only if the relations

imply

$$
\mu \in M(G), \quad \mu * f=0
$$

$$
\mu * g=0 .
$$

In view of the remarks in $(2.2$, iii), it is equivalent to demand that the relations $\mu \in M(G)$ and

$$
\hat{\mu} \cdot \hat{f}=0
$$

$$
\hat{\mu} \cdot \hat{g}=0 .
$$

It is also evident a priori that it is equivalent to demand that the relations $\mu \in L^{1}(G)$ and (3.1.2) imply (3.1.3).

(3.2) In order to use this criterion, it is convenient to introduce the following additional concept and notation. The support $\sigma$ of a pseudomeasure $\sigma$ on $X$ has been defined in $(2.2$, ii). Besides this we now introduce a species of "singular support" of $\sigma$ : let $U$ be the set of points $\xi \in X$ such that $\sigma$ is equal, on some neighbourhood of $\xi$, to a measure; $U$ is evidently an open subset of $X$; the complement $X \cap U^{\prime}$ is here termed the singular support of $\sigma$, denoted by sing $\sigma$. Obviously, sing $\sigma$ is always a closed subset of $\sigma$. (This species of singular support is analogous to, but different from that defined on p. 7 of [10].) If $f \in L^{\infty}(G)$, sing $\hat{f}$ may be termed the singular spectrum of $f$ and denoted by $\operatorname{sing} \Lambda(f)$. 
(3.3) The relation. $t \cdot \sigma=0$. The next step is a discussion of equations of the type (3.1.2) and (3.1.3).

Throughout this subsection, $\sigma$ denotes a pseudomeasure on $X$, and $t$ the FourierStieltjes transform $\hat{\mu}$ of a measure $\mu \in M(G)$; sometimes we shall specialise to the extent of assuming that $\mu \in L^{1}(G)$, i.e., that $t \in A(X)$.

In order that the equation

$$
t \cdot \sigma=0
$$

shall hold, it is obviously necessary that the following condition be fulfilled:

(i) $t=0$ on $\boldsymbol{\sigma}$.

However, (i) is in general not sufficient to imply (3.3.1). We shall therefore record several stronger conditions, each of which will be shown to imply (3.3.1). These reinforced conditions read as follows:

(ia) (i) holds, and furthermore $t=0$ on a neighbourhood of sing $\sigma$.

(ib) $t=0$ on some spectral synthesis set $S$ containing $\sigma[17, \S 7.1 .4$, p. 158$]$.

(ic) (i) holds, $\partial t^{-1}(0) \cap \partial \sigma$ contains no nonvoid perfect set, and either $t \in A(X)$, or $G$ is metrisable.

In (ic) we use $\partial S$ to denote the frontier, relative to $X$, of the subset $S$ of $X$.

The results to be established are these:

(a) (ia) implies (3.3.1).

(b) (ib) implies (3.3.1).

(c) (ic) implies (3.3.1).

Proof of (a). It suffices to show that $t \cdot \sigma=0$ on some neighbourhood of each point $\xi_{0}$ of $X$. This is obviously the case if $\xi_{0} \notin \sigma$, since then $\sigma$ itself vanishes on some neighbourhood of $\xi_{0}$; it is also the case if $\xi_{0} \in \operatorname{sing} \sigma$, thanks to the second clause of (ia).

Consider therefore the case in which $\xi_{0} \in \sigma$ and $\xi_{0} \notin \operatorname{sing} \sigma$. There exists a measure $\lambda \in M(X)$ and a relatively compact neighbourhood $V$ of $\xi_{0}$ such that $\sigma=\lambda$ on $V$, i.e.,

$$
\langle u, \sigma\rangle=\int u d \lambda
$$

for $u \in A(X)$ satisfying $u \subset V$. From this it is easily seen to follow that $\lambda$ vanishes on some neighbourhood of each point of $V \cap \sigma$, and so that $\lambda=0$ on $V \cap \sigma^{\prime}$. (Here and elsewhere, the prime indicates complementation relative to $X$.) Therefore, for each $u \in A(X)$ satisfying $u \subset V$, we have

$$
\begin{aligned}
\langle u, t \cdot \sigma\rangle & =\langle t u, \sigma\rangle=\int t u d \lambda=\int_{V} t u d \lambda \\
& =\int_{V \cap \sigma} t u d \lambda,
\end{aligned}
$$

the last step since $\lambda$ vanishes on $V \cap \sigma^{\prime}$. The final expression here is zero since $t$ vanishes on $\sigma$. Thus $t \cdot \sigma=0$ on $V$, and the proof of (a) is complete. 
Proof of (b). Let $\phi \in L^{\infty}(G)$ be chosen so that $\sigma=\hat{\phi}$. The definition of $\sigma$ leads (by arguments similar to, but simpler than, those in the above proof of (a)) to the conclusion that $u \cdot \sigma=0$ whenever $u \in A(X)$ vanishes on a neighbourhood of $\sigma$, and $a$ fortior if $u$ vanishes on a neighbourhood of $S$. Hence $[17, \S 7.2 .5$ (a), p.161], since $S$ is a spectral synthesis set, it follows that $u \cdot \sigma=0$ for any $u \in A(X)$ which vanishes on $S$. Suppose now that $\left(r_{i}\right)$ is any approximate identity in $L^{1}(G)$, and put $u_{i}=\left(r_{i} * \mu\right)^{\wedge} \in A(X)$. Then $u_{i}$ vanishes on $S$ and so, by what we have just established, $u_{i} \cdot \sigma=0$. On the other hand, $u_{i} \cdot \sigma \rightarrow t \cdot \sigma$ weakly as pseudomeasures. Therefore, taking the limit, we see that $t \cdot \sigma=0$. This proves (b).

Note. It follows from the reference just cited that in fact (i) implies (3.3.1) for all Fourier-Stieltjes transforms $t$ (or, what is equivalent, for all $t \in A(X)$ ), if and only if $\sigma$ is a spectral synthesis set.

Proof of (c). Let $I$ be the closed ideal in $L^{1}(G)$ consisting of those $f \in L^{1}(G)$ such that $\hat{f} \cdot \sigma=0$. Using the notation of $[17, \S 7.1 .3$, p. 158$], Z(I)$ is precisely $\sigma$. So [17, Theorem 7.2.4, pp.160-161], we have $\hat{g} \cdot \sigma=0$ for any $g \in L^{1}(G)$ such that $\partial \hat{g}^{-1}(0) \cap \partial \sigma$ contains no nonvoid perfect set, which finishes the proof in case $t \in A(X)$.

In the remaining case, in which $X$ is $\sigma$-compact, we can take an approximate identity $\left(r_{n}\right)_{n=1}^{\infty}$ in $L^{1}(G)$ such that each $\hat{r}_{n}$ is nonvanishing on $X$. If $t=\hat{\mu}$ with $\mu \in M(G)$, then $g_{n}=r_{n} * \mu \in L^{1}(G), \hat{g}_{n}=\hat{r}_{n} \cdot t$, and $\hat{g}_{n}^{-1}(0)=t^{-1}(0)$. By what has been established already, therefore, $\left(\hat{r}_{n}\right) \cdot \sigma=0$. But $\left(\hat{r}_{n} t\right) \cdot \sigma=\hat{r}_{n} \cdot(t \cdot \sigma) \rightarrow t \cdot \sigma$ weakly as pseudomeasures, and (3.3.1) follows once more.

REMARKS. (i) The last stage of the proof can be carried through whenever either $\partial t^{-1}(0)$ or $\partial \sigma$ is $\sigma$-compact. Possibly it can be done in any case.

(ii) If $t \in A(X)$ the relation (3.3.1) is known to hold provided $t=0$ on $\sigma$ and, in addition, $t$ satisfies suitable conditions of a Lipschitz nature; see [15], Remarks following (6.2), and the references cited there. Such results are of little immediate use since we are at this point not free to impose any such restrictions on $t$, but use will be found for them in (3.6.ii) infra.

It is now a simple matter to derive the following theorem.

(3.4) Theorem. Suppose that $(V, \theta)$ is an admissible pair, and that $f$ and $g$ belong to $V$. Then $g \in T_{\theta}[f]$ provided any one of the following three conditions is fulfilled:

(ia) $\Lambda(g) \subset \Lambda(f)$ and $\sin g \Lambda(g) \subset$ interior $\Lambda(f)$;

(ib) $\Lambda(f)$ contains a spectral synthesis set containing $\Lambda(g)$;

(ic) $\Lambda(g) \subset \Lambda(f)$, and furthermore $\partial \Lambda(g) \cap \partial \Lambda(f)$ contains no nonvoid perfect set.

Proof. It must be shown that (3.1.2) implies (3.1.3), wherein we are lat liberty to suppose that $t=\hat{\mu} \in A(X)$. This will be done by applying $(3.3, \mathrm{a})-(3.3, \mathrm{c})$, taking $\sigma=\hat{g}$, so that $\sigma=\Lambda(g)$. 
Now (3.1.2) entails that $t=0$ on $\hat{f}=\Lambda(f)$, so that the rela tion $\Lambda(g) \subset \Lambda(f)$ entails $(3.3, \mathrm{i})$. Thus (3.3.1) follows, if (ia) or (ib) holds, by virtue of $(3.3, a)$ or $(3.3, b)$ respectively.

In addition to this, if $\xi \in \partial t^{-1}(0)$, then $\xi$ is the limit in $X$ of points $\xi^{*}$ satisfying $t\left(\xi^{*}\right) \neq 0$. For any such point $\xi^{*}$ we have, by (3.1.2), $\xi^{*} \notin \Lambda(f)$. Thus

$$
\partial t^{-1}(0) \subset \overline{\Lambda(f)^{\prime}} .
$$

Also, if $\Lambda(g) \subset \Lambda(f)$, then too $\partial \Lambda(g) \subset \Lambda(g) \subset \Lambda(f)$. This combines with (3.4.1) to yield

$$
\partial t^{-1}(0) \cap \partial \Lambda(g) \subset \overline{\Lambda(f)^{\prime}} \cap \Lambda(f)=\partial \Lambda(f),
$$

and hence also

$$
\partial t^{-1}(0) \cap \partial \Lambda(g) \subset \partial \Lambda(f) \cap \partial \Lambda(g) .
$$

This set contains no nonvoid perfect set, by (ic). Accordingly (3.1.3) follows on the basis of $(3.3, \mathrm{c})$. The proof is complete.

(3.5) Corollary. Suppose $(V, \theta)$ and $f$ are as in (3.4). In order that $T^{*}[f]=V$ it is sufficient that $\Lambda(f)=X$. If in addition $V$ separates points of $M(G)$, and if each $\mu \in M(G)$ defines via (3.1.1) a $\theta$-continuous functional on $V$, this condition is also necessary.

Proof. The sufficiency follows from (3.4). On the other hand, if $\hat{f}$ vanishes on some nonvoid open subset $U$ of $X$, there evidently exists a measure $\mu \in M(G)$ such that $\mu \neq 0$ and $\hat{\boldsymbol{\mu}} \subset U$. Then $\mu$ generates a nonzero $\theta$-continuous functional on $V$ which annihilates $T_{\theta}[f]$ by virtue of the relation $\hat{\mu} \cdot \hat{f}=0$. Thus $T_{\theta}[f]$ cannot exhaust $V$.

(3.6) Remarks. (i) In view of $(3.1, \mathrm{i}),(3.4)$ and (3.5) include as special cases Theorem 4 and its corollary appearing in [6]. If we take $V=B C(G)$ and $\theta=$ the strict topology (see (3.1, ii)) (3.4) shows that $\xi \in X$ is the strict limit of linear combinations of translates of $f$ if and only if $\xi \in \Lambda(f)$.

(ii) It may also be noted that similar arguments lead to the following result about synthesis in the strict topology. Suppose $S$ is a closed subset of $X$ and that $f \in B C(G)$; then $f$ is the strict limit of linear combinations of characters $\xi \in S$ provided $\Lambda(f) \subset S$ and either

$$
\operatorname{sing} \Lambda(f) \subset \text { interior } S,
$$

or $S$ contains a spectral synthesis set which contains $\Lambda(f)$ (which is the case whenever $\partial \Lambda(f)$ contains no nonvoid perfect set; see [17, §7.2.5(b), p. 161].

(iii) If $G=R^{n}$ it is, as has already been stated, possible to refine Lemma (3.3) and its corollaries. The Remarks following (6.2) of [15] show indeed that $t \cdot \sigma=0$ whenever 


$$
t(\xi+\delta) \mathrm{j}=\boldsymbol{O}\left(|\delta|^{n / 2}\right) \text { as } \delta \rightarrow 0,
$$

uniformly for $\xi$ lying in any given compact subset of $\sigma$.

Consider now the linear space $C^{*}\left(R^{n}\right)$ formed of all continuous functions $f$ on $R^{n}$ such that

$$
\lim _{|x| \rightarrow \infty}(1+|x|)^{-n / 2}|f(x)|=0
$$

on $C^{*}\left(R^{n}\right)$ we introduce the norm

$$
\|f\|^{*}=\operatorname{Sup}_{x \in R^{n}}(1+|x|)^{-n / 2}|f(x)|
$$

It is then possible to prove the following result.

Suppose that $f \in C^{*}\left(R^{n}\right)$, that $g \in B C\left(R^{n}\right)$, and that:

$\left.{ }^{*}\right)$ any function $F$ of class $C^{m}\left(R^{n}\right)$, where $m=\left[\frac{1}{2} n\right]$, which vanishes on $\Lambda(f)$ (the support of the Fourier-Schwartz transform of $f$ ), has partial derivatives of orders at most $m$ which vanish on $\Lambda(g)$.

Then $g$ is the limit in $C^{*}\left(R^{n}\right)$ of linear combinations of translates of $f$. If $n=1$, then $m=0$, and $\left(^{*}\right)$ signifies merely that $\Lambda(g) \subset \Lambda(f)$; in this case the preceding result refines in certain respects Theorems 1 and $1^{\prime}$ of [14].

The proof uses the Hahn-Banach theorem; the observation that the dual of $C^{*}\left(R^{n}\right)$ can be identified with the set of measures $\mu$ on $R^{n}$ such that

$$
\int_{R^{n}}(1+|x|)^{n / 2} d|\mu|(x)<\infty ;
$$

and the fact that any such measure $\mu$ has a Fourier-Stieltjes transform $F=\hat{\mu}$ which is of class $C^{m}\left(R^{n}\right)$ and whose partial derivatives of order $m$ satisfy a Lipschitz condition of order $\frac{1}{2} n-\left[\frac{1}{2} n\right]$. As a consequence of this last remark combined with $\left(^{*}\right)$, if $F$ vanishes on $\Lambda(f)$, then

$$
F(\xi+\delta)=O\left(|\delta|^{n / 2}\right) \text { as } \delta \rightarrow 0
$$

uniformly for $\xi \in \Lambda(g)$.

As an immediate corollary it follows that: if $f \in C^{*}\left(R^{n}\right)$ and $\Lambda(f)=R^{n}$, then any $g \in B C\left(R^{n}\right)$ is the limit in $C^{*}\left(R^{n}\right)$ of linear combinations of translates of $f$.

(3.7) Functions $f \in C_{0}(G)$ for which $\operatorname{sing} \hat{f}=X$ have especially interesting translational properties, as will appear from Theorem (3.8) infra. The existence of such functions can be demonstrated, at least whenever $G$ is second countable and noncompact, by use of the category theorem; if $G=R^{n}$, there exist such functions which are further analytic and have all their derivatives in $C_{0}\left(R^{n}\right)$; for details, see [16, Remarks (2.2.5 i) and (2.2.5 ii)]. 
The theorem we aim to establish involves, besides $C_{0}(G)$, the space $Q(G) \subset B U C(G)$ formed of functions $q$ of the type

$$
q(x)=\sum_{\xi \in X} q^{\#}(\xi) \xi(x),
$$

where

$$
\sum_{\xi \in X}\left|q^{\#}(\xi)\right|<\infty .
$$

It is a fact (which we do not use) that $Q(G)$ con sists precisely of the u.a.p. functions on $G$ which are also Fourier-Stieltjes transforms of elements of $M(X)$.

Let us write temporarily $E$ for the vector subspace $C_{0}(G)+Q(G)$ of $B U C(G)$ generated by $C_{0}(G) \cup Q(G)$. By considering the continuous linear map $J$ of $C_{0}(G) \times l^{1}(X)$ into $E$, defined by

$$
J(f, z)=f+\sum_{\xi \in X} z(\xi) \xi
$$

and associating with each continuous linear functional $F$ on $E$ the functional $F \circ J$ on $C_{0}(G) \times l^{1}(X)$, it may be verified that $F$ admits a representation

$$
F(f+q)=\int_{G} f(-x) d \mu(x)+\sum_{\xi \in X} b(\xi) q^{\#}(\xi),
$$

where $\mu \in M(G)$ and $b \in l^{\infty}(X)$ depend on $F$. This paves the way for use of the Hahn-Banach theorem.

As a final preliminary remark, we note that if $f \in C_{0}(G)$ and $\operatorname{sing} \hat{f}=X$, then the only $\mu \in M(G)$ for which $\mu * f$ is a Fourier-Stieltjes transfor m, is $\mu=0$.

We are now ready to state and prove

(3.8) TheOREM. Suppose that $f_{0} \in C_{0}(G)$ is such that sing $\hat{f}_{0}=X$, and that $q_{0} \in Q(G)$. Then $T\left[f_{0}+q_{0}\right]$ contains every function $f+q$, where $f \in C_{0}(G)$, $q \in Q(G)$, and $q^{\#}(\xi)=0$ for every $\xi \in X$ satisfying $q_{0}^{\#}(\xi)=0$. In particular, $T\left[f_{0}+q\right] \supset C_{0}(G)$ for every $q \in Q(G)$.

Proof. Suppose $F$ is a continuous linear functional on $E$ which vanishes on $T\left[f_{0}+q_{0}\right]$ : we must show that $F$ annihilates each function $f+q$ of the stated type. Using (3.7.3), the hypothesis signifies that

$$
\mu * f+\sum_{\xi \in X} b(\xi) q_{0}^{\#}(\xi) \xi=0 .
$$

The second summand on the left-hand side is a Fourier-Stieltjes transform and so, by the closing sentence of (3.7), it follows that $\mu=0$. But then (3.8.1) entails $b(\xi) q_{0}^{\#}(\xi)=0$ for each $\xi \in X$. The desired result therefore follows on using (3.7.3) once again. 


\section{REFERENCES}

1. C. S. Herz, The spectral theory of bounded fuctions, Trans. Amer. Math. Soc. 94 (1960), $181-232$.

2. R. E. Edwards, Functional analysis: Theory and applications, Holt, Rinehart and Winston, New York, 1965.

3. S. Banach, Théorie des opérations linéaires, Monografje Matematyzcne, I, Warsaw, 1932.

4. E. Hewitt, A survey of harmonic analysis, Surveys in Applied Mathematics, Vol. 4, Wiley, New York, 1958.

5. L. H. Loomis, An introduction to abstract harmonic analysis, Van Nostrand, Princeton, N. J., 1953.

6. R. E. Edwards, The exchange formula for distributions and spans of translates, Proc. Amer. Math. Soc. 4 (1953), 888-894.

7. A. Zygmund, Trigonometric series, Vols. I, II, Cambridge Univ. Press, New York, 1959.

8. E. C. Titchmarsh, Introduction to the theory of Fourier integrals, Oxford Univ. Press, Oxford, 1948.

9. L. Schwartz, Théorie des distributions, Tomes I, II, Actualités Sci. Ind. Nos. 1091, 1122, Hermann, Paris, 1950, 1951.

10. L. Hörmander, Linear partial differential operators, Springer, Berlin, 1963.

11. H. Garnir, Les problèmes aux limites de la physique mathématique, Birkhauser Verlag, Basel, 1958.

12. J.-P. Kahane, Transformées de Fourier des functions sommables, Proc. Internat. Congress of Mathematicians, 1962, pp. 114-131, Uppsala, 1963.

13. J.-P. Kahane and R. Salem, Ensembles parfaits et séries trigonométriques, Actualités Sci. Ind. No. 1301, Hermann, Paris, 1963.

14. R. E. Edwards, Approximation theorems for translates, Proc. London Math. Soc. (3) 9 (1959), 321-342.

15. —_ Spans of translates in $L^{p}(G)$, J. Austral. Math. Soc. (2) 5 (1965), 216-233.

16. - - Supports and singular supports of pseudomeasures, J. Austral. Math. Soc. (to appear).

17. W. Rudin, Fourier analysis on groups, Interscience, New York, 1962.

INSTITUTE OF ADVANCED STUdies, AUSTRALian NATIONAL UNIVERSity 